\title{
Deciphering the structure of biological machines at the leading edge of motile cells
}

Dorit Hanein, The Burnham Institute, 10901 North Torrey Pines Road, La Jolla

Californi, 92037.

The actin cytoskeleton provides both the force and the framework for cell motility, adhesion, and intracellular organization. Here at the Burnham Institute (La Jolla, California), my laboratory studies multimolecular complexes involved in the assembly and regulation of the actin cytoskeleton at the leading edge of motile cells. Structural characterization of these multimolecular protein complexes is likely to reveal potential mechanisms underlying cell growth, differentiation and migration, as well as the role of these interactions in tumor invasion and metastasis. The filamentous nature of actin networks precludes crystallographic methods for structure determination. Electron cryomicroscopy has grown to be a powerful imaging technique applicable to almost any kind of specimen, is parsimonious in its material requirements, and allows imaging of large macromolecular assemblies in their fully hydrated state in their native aqueous environment. Our general approach is to combine various electron cryomicroscopy, image analysis and bioinformatic techniques to extract high-resolution structural information. I will describe the role of actin-binding proteins in providing a scaffold for cellular protrusions. 